\title{
Analysis of gluconeogenic and anaplerotic enzymes in Campylobacter jejuni: an essential role for phosphoenolpyruvate carboxykinase
}

\author{
Jyoti Velayudhan and David J. Kelly
}

Author for correspondence: David J. Kelly. Tel: +44 114 224414. Fax: +44 1142728697. e-mail:d.kelly@sheffield.ac.uk

Department of Molecular Biology and Biotechnology, University of Sheffield, Firth Court, Western Bank, Sheffield S10 2TN, UK
Campylobacter jejuni is unable to utilize glucose as a carbon source due to the absence of the key glycolytic enzyme 6-phosphofructokinase. The genome sequence of C. jejuni NCTC 11168 indicates that homologues of all the appropriate enzymes for gluconeogenesis from phosphoenolpyruvate (PEP) are present, in addition to the anaplerotic enzymes pyruvate carboxylase (PYC), phosphoenolpyruvate carboxykinase (PCK) and malic enzyme (MEZ). Surprisingly, a pyruvate kinase (PYK) homologue is also present. To ascertain the role of these enzymes, insertion mutants in pycA, pycB, pyk and mez were generated. However, this could not be acheived for pckA, indicating that PCK is an essential enzyme in $C$. jejuni. The lack of PEP synthase and pyruvate orthophosphate dikinase activities confirmed a unique role for PCK in PEP synthesis. The pycA mutant was unable to grow in defined medium with pyruvate or lactate as the major carbon source, thus indicating an important role for PYC in anaplerosis. Sequence and biochemical data indicate that the PYC of C. jejuni is a member of the $\alpha_{4} \beta_{4}$, acetyl-CoA-independent class of PYCs, with a $65.8 \mathrm{kDa}$ subunit containing the biotin moiety. Whereas growth of the mez mutant was comparable to that of the wild-type, the pyk mutant displayed a decreased growth rate in complex medium. Nevertheless, the mez and pyk mutants were able to grow with pyruvate, lactate or malate as carbon sources in defined medium. PYK was present in cell extracts at a much higher specific activity [ $\left.>800 \mathrm{nmol} \mathrm{min}^{-1}(\mathrm{mg} \text { protein })^{-1}\right]$ than PYC or PCK [ $65 \mathrm{nmol} \mathrm{min}^{-1}$ (mg protein)-1], was activated by fructose 1,6-bisphosphate and displayed other regulatory properties strongly indicative of a catabolic role. It is concluded that PYK may function in the catabolism of unidentified substrates which are metabolized through PEP. In view of the high $K_{\mathrm{m}}$ of MEZ for malate $(\sim 9 \mathrm{mM})$ and the lack of a growth phenotype of the mez mutant, MEZ seems to have only a minor anaplerotic role in $C$. jejuni.

Keywords: pyruvate carboxylase, pyruvate kinase, malic enzyme

\section{INTRODUCTION}

Campylobacter jejuni is a Gram-negative, microaerophilic bacterium recognized as a primary cause of enteric disease worldwide (Tauxe, 1992). Human infection is usually acquired by the consumption of contaminated

Abbreviations: $\mathrm{CAC}$, citric acid cycle; F1,6-BP, fructose 1,6-bisphosphate G6-P, glucose 6-phosphate; MEZ, malic enzyme; OAA, oxaloacetate; PCK, phosphoenolpyruvate carboxykinase; PEP, phosphoenolpyruvate; PYC pyruvate carboxylase, PYK, pyruvate kinase. food, particularly poultry, milk and water. Several factors that contribute to the pathogenesis of C. jejuni have been identified, including motility, adhesion, the ability to invade host cells and the production of several toxins (Ketley, 1997). Although the organism can clearly adapt its metabolism to a range of different environments, within and outside of the host, the factors that contribute to its metabolic versatility are poorly defined.

The complete genome sequence of C. jejuni NCTC 11168 (Parkhill et al., 2000) has provided numerous insights into the metabolism and physiology of this 


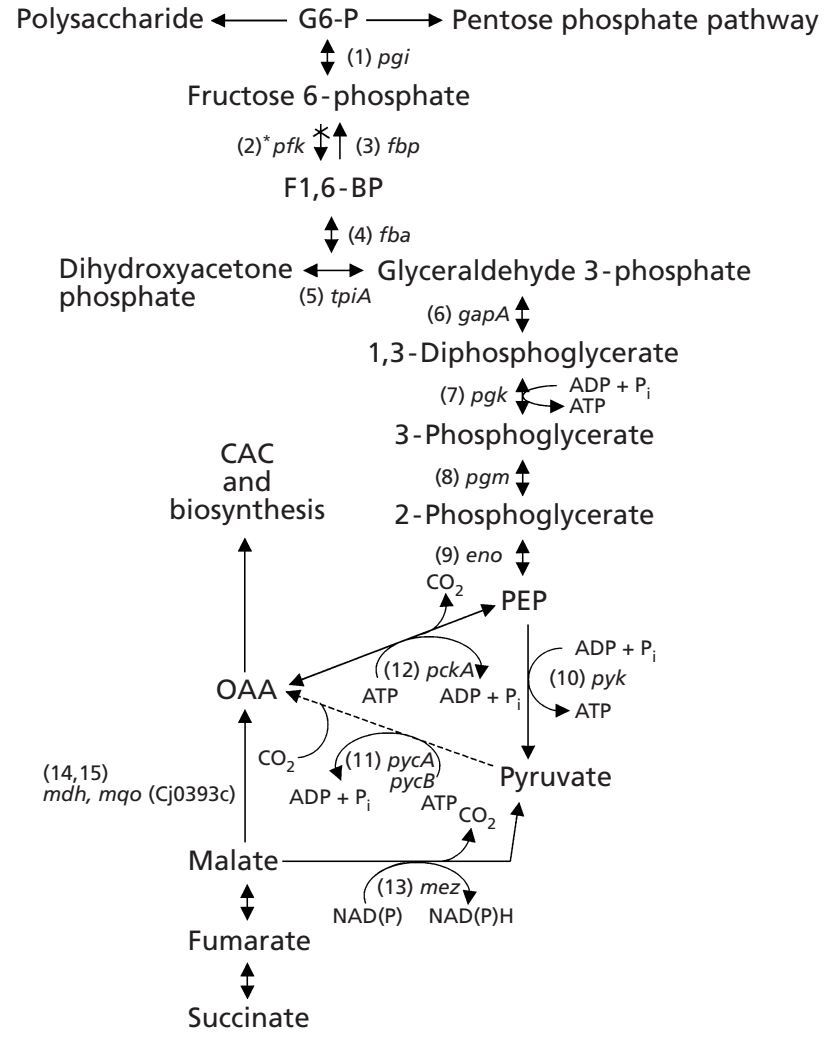

Fig. 1. Outline of the gluconeogenic and anaplerotic reactions of C. jejuni, based on genome sequence data and biochemical data. The broken arrow highlights the CAC-replenishing anaplerotic reaction of PYC. Numbers indicate genes for the following enzymes: 1, glucose-6-phosphate isomerase; 2, 6phosphofructokinase, the asterisk indicates that the $C$. jejuni genome lacks a homologue for the 6-phosphofructokinase, which catalyses the irreversible phosphorylation of fructose 6phosphate to F1,6-BP in glycolysis; 3, fructose-1,6-bisphosphatase; 4, fructose-bisphosphate aldolase; 5, triosephosphate isomerase; 6, glyceraldehyde-3-phosphate dehydrogenase; 7, phosphoglycerate kinase; 8 , phosphoglycerate mutase; 9, enolase; 10, PYK; 11, PYC; 12, PCK; 13, MEZ; 14, malate dehydrogenase; 15, malate:quinone oxidoreductase, possibly encoded by Cj0393c.

organism. Although it is widely accepted that C. jejuni neither oxidizes nor ferments glucose (Smibert, 1984), genes encoding enzymes of the Embden-Meyerhof and pentose phosphate pathways are present in the genome (Parkhill et al., 2000). Most of the reactions of the Embden-Meyerhof pathway are reversible in vivo, with the notable exceptions of the 6-phosphofructokinase and pyruvate kinase (PYK) reactions (Fig. 1), both of which constitute key control points. C. jejuni lacks a gene that could encode a 6-phosphofructokinase, which may account for the inability of the bacterium to catabolize glucose. However, a gene encoding fructose1,6-bisphosphatase is present (Fig. 1), which strongly suggests that the Embden-Meyerhof pathway functions in gluconeogenesis. Nevertheless, a homologue of PYK (Cj0392c) has also been identified (Parkhill et al., 2000). This is surprising, since PYK catalyses the physio- logically irreversible conversion of phosphoenolpyruvate (PEP) and ADP to pyruvate and ATP at the final stage of the glycolytic pathway (Fig. 1).

C. jejuni is predicted to possess a complete citric acid cycle (CAC) (Parkhill et al., 2000; Kelly, 2001). The net synthesis of oxaloacetate (OAA) from pyruvate or PEP is required to permit the CAC to fulfil both its biosynthetic and its energy-generating roles. C. jejuni possesses homologues of anaplerotic enzymes that may function in this capacity. These are as follows. (i) Pyruvate carboxylase (PYC) encoded by pycA (Cj1037c) and $p y c B$ (Cj0933c) homologues, which correspond to the biotin carboxylase and biotin carboxyl carrier subunits, respectively, of the two-subunit type of carboxylase (Goss et al., 1981). PYC is a biotin-containing enzyme that fulfils an important anaplerotic function in many organisms by catalysing the ATP-dependent carboxylation of pyruvate to yield OAA (Fig. 1). (ii) Phosphoenolpyruvate carboxykinase (PCK) which provides the PEP required for gluconeogenesis (Fig. 1). PCK catalyses the ATP-dependent decarboxylation of OAA to PEP. C. jejuni has a homologue of the Escherichia coli pckA gene (Cj0932c) and, interestingly, it is contiguous with the $p y c B$ homologue. (iii) Besides PYC and PCK, the only other predicted decarboxylating/ carboxylating enzyme located at the metabolic node around pyruvate is malate oxidoreductase [malic enzyme (MEZ)]. MEZ catalyses the oxidative decarboxylation of malate to pyruvate coupled with $\operatorname{NAD}(\mathrm{P})$ reduction (Fig. 1). Cj1287c encodes a probable malate oxidoreductase, homologous to that of Bacillus stearothermophilus (Parkhill et al., 2000).

Given the likely gluconeogenic role of the EmbdenMeyerhof pathway in C. jejuni, the presence and role of PYK is particularly enigmatic, as a futile cycle could result from the PYC, PCK and PYK reactions (Fig. 1). This study was undertaken to investigate the physiological function of the anaplerotic enzymes in C. jejuni and to determine the mode of regulation of the PYK. The phenotypic consequences of the mutational inactivation of PYK and the anaplerotic enzymes in $C$. jejuni are reported, permitting the assignment of definitive functions to several genes identified in the complete genome sequence (Parkhill et al., 2000).

\section{METHODS}

Bacterial strains, media and culture conditions. C. jejuni strains were routinely grown at $37^{\circ} \mathrm{C}$ under microaerobic conditions $\left[10 \%(\mathrm{v} / \mathrm{v}) \mathrm{O}_{2}, 5 \%(\mathrm{v} / \mathrm{v}) \mathrm{CO}_{2}\right.$ and $\left.85 \%(\mathrm{v} / \mathrm{v}) \mathrm{N}_{2}\right]$ in a MACS-VA500 cabinet (Don Whitley Scientific) on Columbia agar containing $5 \%(\mathrm{v} / \mathrm{v})$ lysed horse blood and $10 \mu \mathrm{g} \mathrm{ml}^{-1}$ each of amphotericin B and vancomycin. Liquid cultures of C. jejuni were grown with gentle agitation under the same conditions, in brain heart infusion (BHI) broth supplemented with $5 \%(\mathrm{v} / \mathrm{v})$ fetal calf serum or in the defined medium MEM $\alpha$ (Gibco), both containing the above antibiotics. MEM $\alpha$ is composed of a mixture of inorganic salts, amino acids, vitamins, ribonucleosides and deoxyribonucleosides. To select for C. jejuni mutants carrying antibioticresistant determinants, either kanamycin or chloramphenicol 
Table 1. Oligonucleotide primers used in this study

\begin{tabular}{|lll|}
\hline Primer & \multicolumn{1}{c|}{ Annealing site } & \multicolumn{1}{c|}{ Primer sequence $\left(\mathbf{5}^{\prime} \mathbf{- \mathbf { 3 } ^ { \prime }}\right)$} \\
\hline PYCA-F & ORF Cj1037c, bases $46-71$ & ATGCGCTGTTAGAGTAATCCGTGCTTGTAG \\
PYCA-R & ORF Cj1037c, bases 1394-1419 & ATGCAAGCGTAGCAGCTATTGCAGCTATAA \\
PYCB-F & ORF Cj0933c, bases 1-23 & ATGCCATATGGCTAAAAAATTCATCGATGT \\
PYCB-R & ORF Cj0933c, bases 1771-1797 & ATGCATTCTTGTAAATAGCTAATACTTCGC \\
PCKA-F & ORF Cj0932c, bases 137-162 & ATGCCAGGGATTTTTACAGGTAGAAGTCCT \\
PCKA-R & ORF Cj0932c, bases 1540-1565 & ATGCGGTCCAAATTTGCTAAATTCAATGCC \\
PYK-F & ORF Cj0392c, bases 33-58 & ATGCAGGTCCAGCAAGTGAAAAGGAAGAAA \\
PYK-R & ORF Cj0392c, bases 1387-1412 & ATGCTTGAGAATGCGGATAAGATTGCTAGT \\
MEZ-F & ORF Cj1287c, bases $-102--79$ & ATGCGGAGTGCGGTAAGTCCTAGTATTT \\
MEZ-R & ORF Cj1287c, bases 1161-1184 & ATGCACACCATCTTTTAGCGCAGCTGCT \\
\hline
\end{tabular}

was added to a final concentration of $30 \mu \mathrm{g} \mathrm{ml}^{-1}$. E. coli DH5 $\alpha$ was cultured at $37^{\circ} \mathrm{C}$ in Luria-Bertani (LB) medium supplemented with appropriate antibiotics.

For growth assays, C. jejuni overnight starter cultures were prepared in BHI broth before inoculation into fresh BHI or $\operatorname{MEM} \alpha$ medium to which selected carbon sources at specified concentrations were added. Growth was monitored at $600 \mathrm{~nm}$ in an Amersham Pharmacia Biotech Ultrospec 2000 spectrophotometer.

DNA isolation and manipulation. Plasmid DNA was isolated using the Qiagen Miniprep kit. C. jejuni chromosomal DNA was extracted using a modified SDS lysis procedure (Marmur, 1961). Standard techniques were employed for the cloning, transformation, preparation and restriction analysis of plasmid DNA from E. coli (Sambrook et al., 1989).

Preparation of cell extracts and enzyme assays. Cell-free extracts were prepared from $300 \mathrm{ml}$ cultures grown to stationary phase. Cells were harvested in a Beckman Avanti $\mathrm{J}$-25 I centrifuge $\left(20 \mathrm{~min}, 4^{\circ} \mathrm{C}, 8000 \mathrm{~g}\right)$, washed with $0 \cdot 1 \mathrm{M}$ Tris $/ \mathrm{HCl}(\mathrm{pH} 8.0)$ and then resuspended in $1 \mathrm{ml}$ of the same buffer before sonication (MSE Soniprep 150, $6 \times 10 \mathrm{~s}$ bursts, $12 \mu \mathrm{m}$ amplitude). The crude extract was clarified by centrifugation $(30 \mathrm{~min}, 14000 \mathrm{~g}$ ) and the supernatant was retained for use on the same day.

PYC and PCK activities were monitored in assays linked to the NADH-dependent reduction of OAA by malate dehydrogenase. Each $1 \mathrm{ml}$ PYC assay mixture contained $0 \cdot 1 \mathrm{M}$ Tris $/ \mathrm{HCl}(\mathrm{pH} 8.0), 5 \mathrm{mM}$ sodium pyruvate, $5 \mathrm{mM}$ ATP, $50 \mathrm{mM} \mathrm{NaHCO}_{3}, 5 \mathrm{mM} \mathrm{MgCl}_{2}, 0 \cdot 25 \mathrm{mM} \mathrm{NADH}, 2 \mathrm{U}$ malate dehydrogenase (Sigma) and cell extract. The reaction was initiated by the addition of pyruvate, and the disappearance of NADH was monitored continuously at $340 \mathrm{~nm}$. PCK activity was measured in $1 \mathrm{ml}$ assays containing $100 \mathrm{mM}$ MOPS (pH 7.0), $50 \mathrm{mM} \mathrm{NaHCO}, 4 \mathrm{mM}$ ADP, $2.5 \mathrm{mM} \mathrm{MgCl}$, $2 \mathrm{mM} \mathrm{MnCl}_{2}, 0 \cdot 25 \mathrm{mM}$ NADH, $2 \mathrm{mM}$ PEP, $2 \mathrm{U}$ malate dehydrogenase and cell extract. MEZ activity was assayed in a $1 \mathrm{ml}$ mixture containing $0 \cdot 1 \mathrm{M}$ Tris $/ \mathrm{HCl}(\mathrm{pH} 7 \cdot 0), 2 \mathrm{mM}$ $\mathrm{MnCl}_{2}, 5 \mathrm{mM} \mathrm{KCl}, 2 \cdot 5 \mathrm{mM}$ NADP, $7 \cdot 5 \mathrm{mM}$ L-malate and cell extract. PYK activity was assayed in a mixture containing $0 \cdot 1 \mathrm{M}$ Tris $/ \mathrm{HCl}(\mathrm{pH} 7 \cdot 0), 30 \mathrm{mM} \mathrm{MgSO}, 10 \mathrm{mM}$ ADP, $0.25 \mathrm{mM}$ NADH, $5 \mathrm{mM}$ PEP, $5 \mathrm{U}$ lactate dehydrogenase (Sigma) and cell extract. The $K_{\mathrm{m}}, V_{\max }$ and Hill coefficients of the enzymes were estimated by using the curve-fitting package Enzfitter from Biosoft.

Detection of biotinylated proteins. Cell extracts from wildtype and $p y c$ mutant cells, grown in BHI broth, were prepared as described above. Denatured extracted protein $(0.3 \mathrm{mg})$ was loaded and separated by SDS-PAGE $(12 \%, \mathrm{w} / \mathrm{v}$, acrylamide), then electroblotted onto a PVDF membrane (Millipore) in a Bio-Rad Transblot cell at $250 \mathrm{~mA}$ for $16 \mathrm{~h}$. Biotinylated polypeptides were detected using an avidin-horseradish peroxidase conjugate (Bio-Rad), according to the instructions of the manufacturer.

Insertional inactivation of the $p y c A, p y c B, p y k, p c k A$ and $m e z$ genes. Primers PYCA-F and PYCA-R (Table 1) were designed to amplify a $1.4 \mathrm{~kb}$ fragment of the $p y c A$ homologue $(\mathrm{Cj} 1037 \mathrm{c})$ by PCR. This fragment was subsequently cloned into pGEMT Easy (Promega). The aphA-3-containing SmaI fragment of pJMK30 (van Vliet et al., 1998), which confers kanamycin resistance, was then cloned into the BglII site within the Cj1037c gene to generate pJV1. The C. jejuni pycB homologue (Cj0933c) was amplified with the primers PYCB-F and PYCB-R (Table 1) and cloned into pGEM-T Easy. A chloramphenicol-acetyltransferase (CAT) cassette, originating from Campylobacter coli (Wang \& Taylor, 1990), was then cloned into a unique BstXI site located within the cloned $p y c B$ gene to generate $\mathrm{pJV} 2$. For the inactivation of the $p c k A$ homologue (Cj0932c), primers PCKA-F and PCKA-R (Table 1) were used to amplify a $1.4 \mathrm{~kb}$ fragment of Cj0932c from chromosomal DNA and the resulting amplicon was cloned into pGEM-T Easy. The CAT cassette was then cloned into a unique BstEII site within the cloned Cj0932c fragment, yielding pJV3. A fragment of the pyk homologue (Cj0392c) was amplified with PYK-F and PYK-R (Table 1). The resulting amplicon was cloned into pGEM-T Easy and the CAT cassette was inserted into the unique $K p n \mathrm{I}$ site within the cloned $p y k$ gene; this construct was named pJV4.

For the inactivation of the putative MEZ gene (here designated mez) primers MEZ-F and MEZ-R (Table 1) were used to amplify a fragment of Cj1287c. The $1.4 \mathrm{~kb}$ fragment was cloned into pGEM-T Easy and the aphA-3 gene was then ligated to the unique $B s m \mathrm{I}$ site within the cloned fragment, producing pJV5. Except where indicated, all constructs contained the antibiotic-resistance cartridge in the same transcriptional orientation as that of the gene (Fig. $2 a-d)$.

All antibiotic-resistant constructs were introduced into $C$. jejuni NCTC 11168 by electroporation (Ferrero et al., 1992). Transformants were selected on Columbia agar plates supplemented with the appropriate antibiotic. Single colonies were restreaked and chromosomal DNA was extracted by the microLYSIS kit (Web Scientific) for screening by PCR, to ensure that allelic exchange had occurred by double-homologous recombination. 
(a)

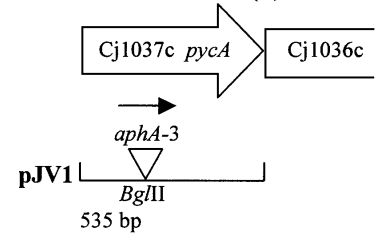

(c)
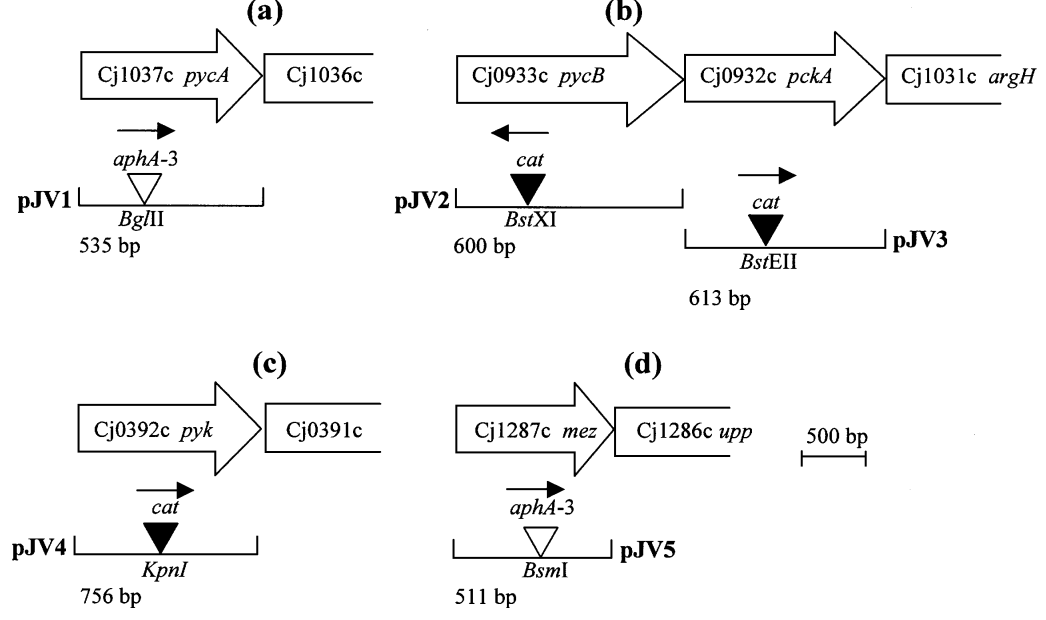

(d)

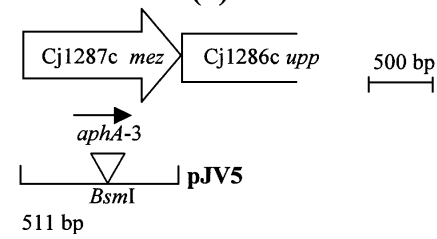

(e)

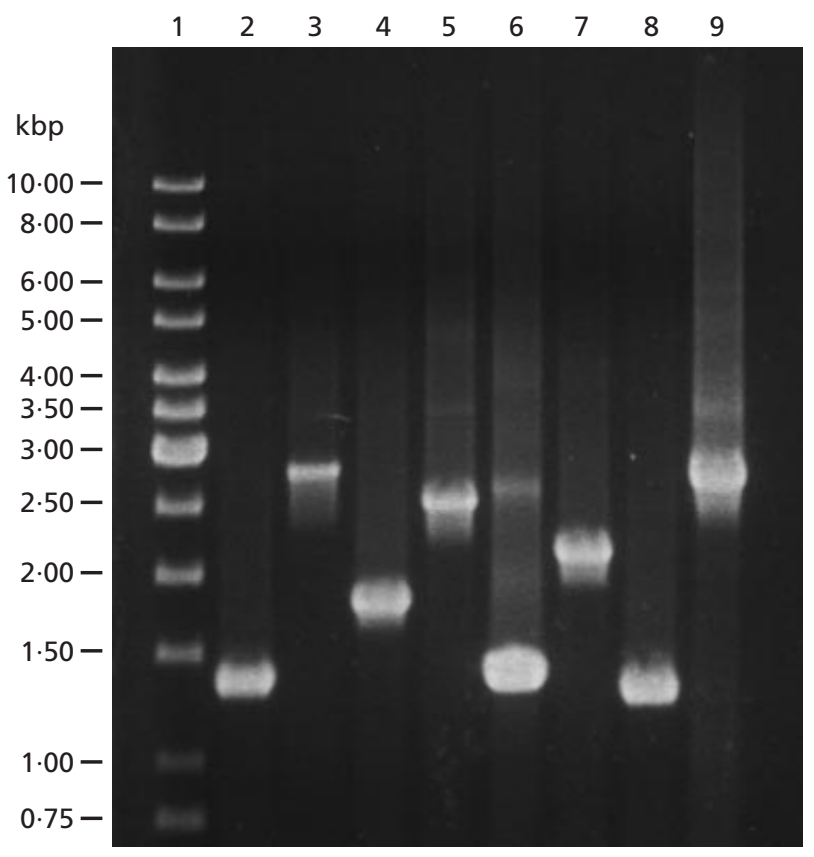

Fig. 2. Organization and mutagenesis strategy of the pycA (a), pycB pckA (b), pyk (c) and mez (d) genes in C. jejuni NCTC 11168 and mutant verification (e). Mutants were constructed by the insertion of cat or aphA-3 cassettes (inverted triangles) into unique restriction sites within each gene to give the constructs shown. The distance from the translational start site of the gene to the cassette insertion site is indicated. The cassettes employed lack the $3^{\prime}$ transcriptional termination signal and were inserted with the same polarity as the pycA, pckA, pyk and mez genes (shown by an arrow above the cassette), whereas the $p y c B$ gene contained the cat cassette in the reverse orientation. (e) Agarose gel $(0.7 \%)$ of PCR products obtained with the parental strain, C. jejuni NCTC 11168, genomic DNA as template (lanes 2, 4, 6, 8) or with DNA from pycA (lane 3), pycB (lane 5), pyk (lane 7) and mez (lane 9) mutants, using primers PYCA-F and PYCA-R (lanes 2 and 3), PYCB-F and PYCB-R (lanes 4 and 5), PYK-F and PYK$R$ (lanes 6 and 7) or MEZ-F and MEZ-R (lanes 8 and 9). Lane 1 contains a $1 \mathrm{~kb}$ DNA ladder. The size increase of the PCR products obtained when mutant DNA was used as the template corresponds to the 0.75 and $1.5 \mathrm{~kb}$ cat and aphA-3 cassettes, respectively.

\section{RESULTS}

Inactivation of the chromosomal pycA, pycB, pyk,
pckA and mez genes

As a first step towards defining roles for PYC, PYK, PCK and MEZ, mutants were constructed by the insertion of non-polar antibiotic-resistance cassettes (containing an internal promoter, but no transcriptional terminators) into unique restriction sites within the cloned genes to give pJV1-pJV5 (Fig. 2). These constructs were electroporated into C. jejuni NCTC 11168 , generating chromosomal null mutants of $p y c A, p y c B, p y k$ and the mez gene by homologous recombination. The correct construction of the mutants was confirmed by PCR amplification of the target genes, which yielded fragment sizes consistent with the insertion of the 0.75 and $1.5 \mathrm{~kb}$ chloramphenicol- and kanamycin-resistance cassettes, respectively (Fig. 2). Despite numerous attempts, no chloramphenicol-resistant colonies were obtained with pJV3. As other transformations performed in parallel were successful, this indicates that $p c k A$ is an essential gene. All of the genes that were successfully inactivated contained the antibiotic-resistance determinant in the same transcriptional orientation as that of the target gene, with the exception of $p y c B$. Mutants of $p y c B$ could only be obtained with constructs containing the antibiotic-resistance cassette in the opposite transcriptional orientation to that of the gene. It is likely that this requirement for a specific orientation is due to polar effects on the level of expression of the downstream $p c k A$ (see below). Insertion of the antibiotic-resistance cassette in the same direction as that of the $p y c B$ gene may result in a level of PCK which is superoptimal and, therefore, detrimental to growth. Conversely, as shown in the following section, the presence of the antibioticresistance cassette in the opposite orientation in $p y c B$ 
Table 2. Activities of selected enzymes in the $C$. jejuni wild-type, pycA, pycB, pyk and mez mutant strains

Enzyme specific activities were determined in stationary-phase cultures grown in BHI broth. The values represent the mean \pm SD of two independent experiments, each performed in duplicate.

\begin{tabular}{|lcccc|}
\hline Strain & \multicolumn{3}{c|}{ Specific activity $\left[\mathbf{n m o l ~ \mathbf { ~ m i n } ^ { - 1 } ( \mathbf { m g } \text { protein } ) ^ { - 1 } ]}\right.$} \\
\cline { 2 - 5 } & PYC & PYK & PCK & MEZ \\
\hline NCTC 11168 wild-type & $47 \pm 3$ & $811 \pm 9$ & $61 \pm 1$ & $86 \pm 10$ \\
pycA mutant & 0 & $634 \pm 75$ & $56 \pm 4$ & $13 \pm 1$ \\
pycB mutant & 0 & $495 \pm 20$ & $7 \pm 3$ & $87 \pm 15$ \\
pyk mutant & $51 \pm 4$ & 0 & $18 \pm 1$ & $106 \pm 15$ \\
mez mutant & $30 \pm 2$ & $800 \pm 21$ & $53 \pm 6$ & 0 \\
\hline
\end{tabular}

still resulted in weak PCK activity, sufficient to support growth of the $p y c B$ mutant.

\section{Enzymic properties of the pycA, pycB, pyk and mez mutants}

The wild-type strain and the mutants were assayed for their PYC, PYK, PCK and MEZ activities, to investigate whether mutations in the $p y k, p y c A, p y c B$ and $m e z$ genes affected enzyme activity at the metabolic junction around pyruvate. The results are displayed in Table 2. PYC activity was completely abolished in the $p y c A$ and $p y c B$ mutants. PYK activity was reduced somewhat in the $p y c$ mutants, with this effect being more pronounced with the $p y c B$ mutant (approximately twofold lower than that of the wild-type). Whereas PCK activity was not altered in the $p y c A$ mutant, the $p y c B$ mutant displayed an approximately ninefold reduction in PCK activity. This indicates that the $p y c B$ mutation is polar, affecting the expression of $p c k A$ (which lies $13 \mathrm{bp}$ downstream of the $p y c B$ gene). MEZ activity was reduced fourfold in the $p y c A$ mutant, although MEZ activity in the $p y c B$ mutant was similar to that of the wild-type. The reason for this discrepancy is unclear. As shown in Table 2, the $p y k$ mutant was devoid of PYK activity. Although MEZ and PYC activities in the $p y k$ mutant were comparable to those in the wild-type, PCK activity was reduced by approximately threefold. It should be noted that although PEP and ADP are substrates for the PYK reaction they are also substrates for PCK in PEP carboxylation (Fig. 1), but the latter reaction also requires bicarbonate. Hence, the PYK assay is specific and this is confirmed by the lack of any residual activity in the $p y k$ mutant. The possibility that PYK activity interferes with the PCK assay is precluded by the fact that the pyk mutant displays decreased (not increased) PCK activity. MEZ activity was abolished in the mez mutant (Table 2), thus confirming the identity of Cj1287c as the gene that encodes MEZ. PYC, PYK and PCK activities remained largely unaltered in this strain. Extracts of the wild-type strain were devoid of pyruvate orthophosphate dikinase activity, which catalyses the reversible, ATP-dependent conversion of pyruvate to PEP. Furthermore, no PEP synthase activity was detected in wild-type cell extracts. PEP synthase catalyses the conversion of pyruvate and ATP to PEP, AMP and phosphate, and is thought to function in gluconeogenesis. Thus, PCK appears to be the only means of synthesizing PEP, which is consistent with the failure to isolate a $p c k A$ mutant.

\section{Growth characteristics of the pycA, pycB, pyk and mez mutants}

The effect of mutations in PYK and in the anaplerotic enzymes on the growth of C. jejuni NCTC 11168 was determined in BHI broth (Fig. 3). Doubling times for the wild-type strain and the $p y c A$ mutant were almost identical ( $\sim 2$ and $\sim 3 \mathrm{~h}$, respectively), with their final growth yields being similar (Fig. 3a). The $p y c B$ mutant, however, displayed a significant reduction in both growth rate and yield, with a doubling time of $\sim 7 \mathrm{~h}$ and an approximately threefold reduction in the final extent of growth (Fig. 3a). Moreover, supplementation of the growth medium with malate did not rescue the growth defect of the $p y c B$ mutant (data not shown). The pyk mutant exhibited a decreased growth rate compared to that of the wild-type, with a doubling time of $\sim 5 \mathrm{~h}$, although the final extent of growth was not affected (Fig. $3 b$ ). The addition of pyruvate to the medium did not enhance the growth rate of the pyk mutant (data not shown). Growth of the mez mutant, with respect to both the growth rate and the final cell yields, was comparable to that of the wild-type (Fig. 3c).

To determine the carbon sources capable of supporting the growth of the wild-type and the mutant strains, growth assays were repeated using the defined medium $M E M \alpha$. Growth of the $p y c A$ mutant and the parent strains in MEM $\alpha$ supplemented with either pyruvate, lactate or malate showed that the mutant was severely impaired in its growth when pyruvate or lactate was the major carbon source, although its growth on malate was not affected (Fig. 4a). The $p y c B$ mutant, on the other hand, was unable to grow on lactate, pyruvate or malate (Fig. 4a). These results indicate that the PYC in C. jejuni contributes significantly to growth on lactate and on pyruvate. To test the hypothesis that a depletion in intracellular OAA is responsible for the deficient growth 

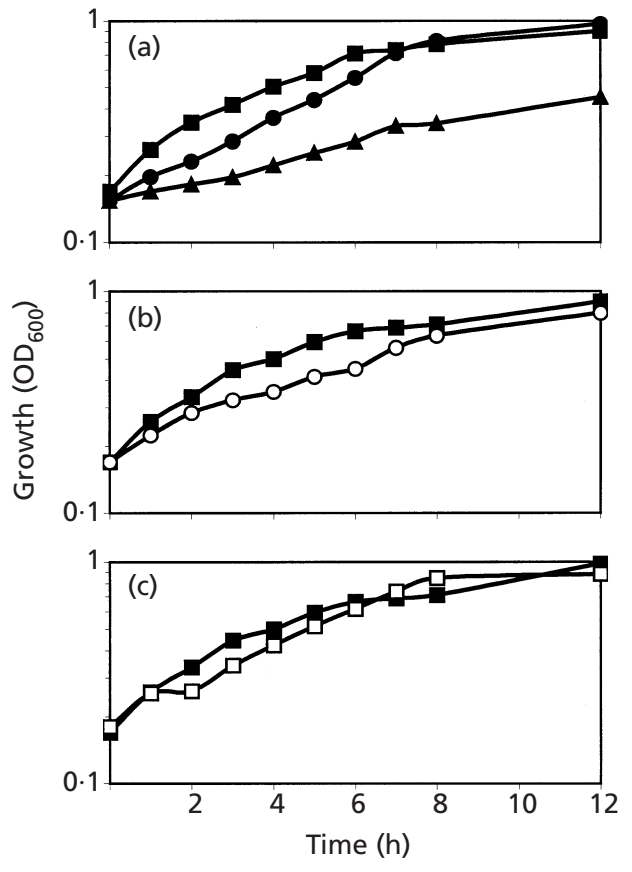

Fig. 3. Comparative growth of the $C$. jejuni wild-type and mutant strains. (a) $\mathbf{\square}, C$. jejuni wild-type;, pycA mutant; $\boldsymbol{\Delta}$, pycB mutant. (b) $\mathbf{\square}, C$. jejuni wild-type; $\bigcirc$, pyk mutant. (c) $\mathbf{\square}$, C. jejuni wild-type; $\square$, mez mutant . C. jejuni starter cultures were grown overnight in $\mathrm{BHI}$ broth and then inoculated into fresh, prewarmed $\mathrm{BHI}$ broth. The data are from individual growth assays, but are representative of two independent experiments.

of the $p y c A$ mutant on pyruvate and on lactate, a small amount $(1 \mathrm{mM})$ of malate was added to a $p y c A$ mutant culture, with lactate $(20 \mathrm{mM})$ as the major carbon source. The addition of malate restored the final cell density of the $p y c A$ mutant to the wild-type level (Fig. $4 \mathrm{~b})$. The addition of the same concentration of malate to the wild-type had no effect on its growth (Fig. 4b). The

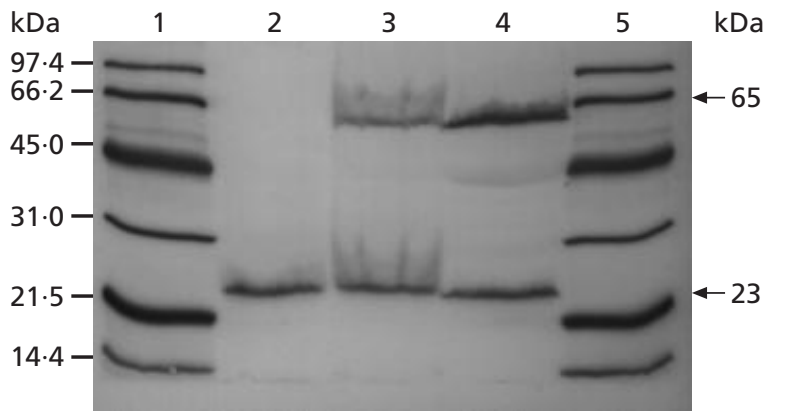

Fig. 5. Detection of biotinylated polypeptides in wild-type cell extracts and in pyc $A$ and $p y c B$ mutant cell extracts. The denatured proteins were separated in a $12 \%$ polyacrylamide gel. The amount of cell extract protein per lane was $0.3 \mathrm{mg}$. The biotin-containing polypeptide bands were detected using an avidin-horseradish peroxidase conjugate. Lanes: 1 and 5, biotinylated molecular mass markers, phosphorylase $B, 97.4 \mathrm{kDa}$, bovine serum albumin, $66.2 \mathrm{kDa}$, ovalbumin, $45.0 \mathrm{kDa}$, carbonic anhydrase, $31.0 \mathrm{kDa}$, soybean trypsin inhibitor, $21.5 \mathrm{kDa}$, lysosyme, $14.4 \mathrm{kDa} ; 2$, pycB mutant; 3, pycA mutant; 4, C. jejuni wild-type.

inability of the $p y c B$ mutant to utilize malate as a carbon source is almost certainly a result of the marked reduction in PCK activity, thus indicating the importance of PCK during growth on malate. Growth of the pyk and the mez mutants on all three carbon sources was comparable to that of the wild-type (Fig. 4a).

\section{Synthesis of the biotinylated PYCB protein in the pycA mutant}

Several carboxylases, most notably pyruvate carboxylase and acetyl-CoA carboxylase, possess covalently bound biotin at their active sites. Western blots of $C$. jejuni NCTC 11168 cytosolic proteins probed with avidin-horseradish peroxidase revealed at least two biotinylated polypeptides of approximately 65 and $23 \mathrm{kDa}$ (Fig. 5), the former being the most abundant.
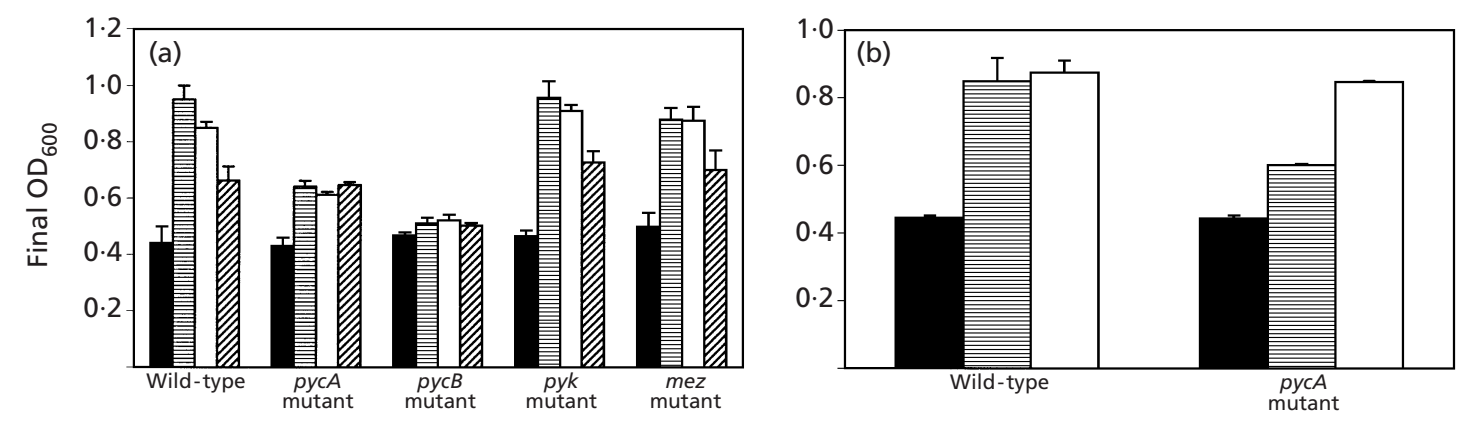

Fig. 4. (a) Growth of $C$. jejuni wild-type, pycA, pycB, pyk and mez mutant strains in MEM $\alpha$, in the presence and absence of an added carbon source. Solid bars, no added carbon source; horizontal hatched bars, pyruvate (20 mM); open bars, lactate $(20 \mathrm{mM})$; hatched bars, malate $(20 \mathrm{mM})$. The data represent the mean of two separate experiments \pm SD. (b) Growth of C. jejuni wild-type and pycA mutant strains in MEM $\alpha$ supplemented with $20 \mathrm{mM}$ lactate plus or minus 1 mM malate. Solid bars, no added carbon source; horizontal hatched bars, $20 \mathrm{mM}$ lactate alone; open bars, $20 \mathrm{mM}$ lactate plus $1 \mathrm{mM}$ malate. The data represent the mean of two independent experiments \pm SD. Strains were pre-grown overnight in $\mathrm{BHI}$ broth, harvested, washed and resuspended in MEM $\alpha$ before inoculating into prewarmed MEM $\alpha$ broth. It should be noted that in $\operatorname{MEM} \alpha$ alone a basal level of growth occurs, due to the presence of amino acids in the medium (starting $\left.\mathrm{OD}_{600} 0 \cdot 25\right)$. 

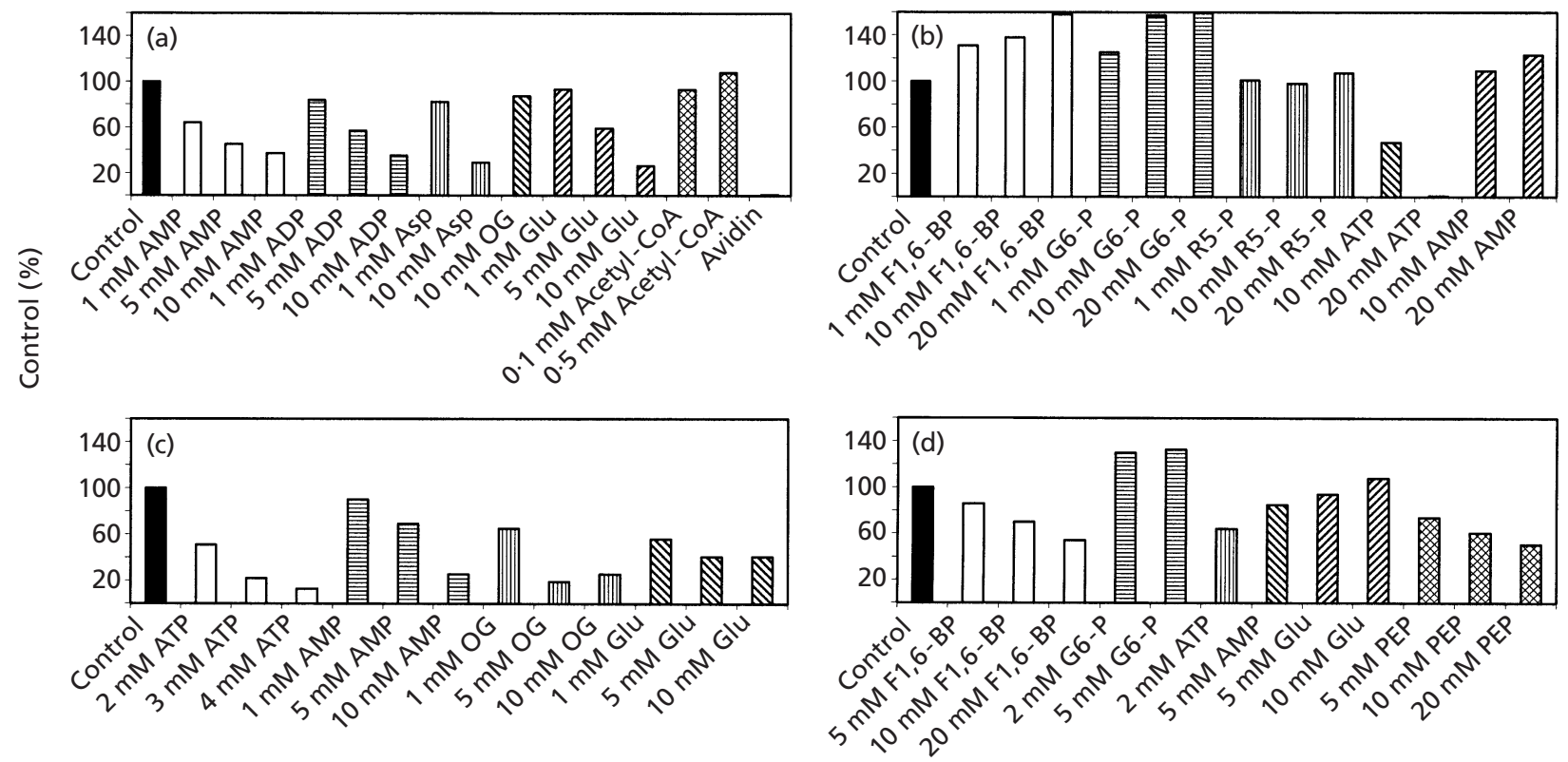

Effector

Fig. 6. Influence of effectors, at various concentrations, on PYC (a), PYK (b), PCK (c) and MEZ (d) activities in wild-type cell extracts. The indicated activities were normalized to the activity in the absence of any effector. Control activities were $51 \mathrm{nmol} \mathrm{min}^{-1}$ (mg protein) $)^{-1}$ for PYC, $788 \mathrm{nmol} \mathrm{min}^{-1}\left(\mathrm{mg}\right.$ protein) ${ }^{-1}$ for PYK, $59 \mathrm{nmol} \mathrm{min}^{-1}$ (mg protein) ${ }^{-1}$ for PCK and $54 \mathrm{nmol} \mathrm{min}^{-1}$ (mg protein) ${ }^{-1}$ for MEZ. OG, 2-oxoglutarate; R5-P, ribose 5-phosphate.

This is likely to be the PYCB protein, the predicted molecular mass of which is $65 \cdot 8 \mathrm{kDa}$. The $p y c B$ mutant was specifically deficient in the $65 \mathrm{kDa}$ protein, thus establishing its identity as the biotinylated subunit of PYC. The pycA mutant, on the other hand, shared the same biotinylated-protein profile with the wild-type, suggesting that although PYCB is still synthesized, the lack of PYCA prevents the assembly of a functional protein. The $23 \mathrm{kDa}$ protein is similar in size to the biotin carboxyl carrier proteins of acetyl-CoA carboxylases from E. coli, Anabaena sp. strain PCC 7120 and Pseudomonas aeruginosa (Li \& Cronan, 1992; Gornicki et al., 1993; Best \& Knauf, 1993).

\section{Biochemical characteristics of anaplerotic enzymes in wild-type cells}

The activity of PYC was strictly dependent on the presence of ATP, pyruvate, bicarbonate and $\mathrm{Mg}^{2+}$, although the requirement for the presence of a divalent cation could be fulfilled by $\mathrm{Co}^{2+}$ and poorly by $\mathrm{Mn}^{2+}$. PEP could not substitute for pyruvate and ATP could not be replaced with ADP or AMP. In the presence of ATP, both AMP and ADP acted as potent inhibitors (Fig. 6a). Similar to other PYCs, ATP inhibited the enzyme when present at concentrations above $7 \mathrm{mM}$. The inhibition caused by ATP was relieved by the presence of excess $\mathrm{Mg}^{2+}$. Incubation of the cell extract with avidin completely abolished PYC activity (Fig. 6a). PYC activity was not affected by acetyl-CoA (Fig. 6a). Although insensitive to 2-oxoglutarate, PYC was significantly inhibited by glutamate and aspartate (Fig. 6a).
Michaelis-Menten kinetics were followed when the initial velocity was plotted against varying pyruvate and bicarbonate concentrations (Table 3).

The PYK of C. jejuni exhibited classical saturation kinetics with ADP, whereas the enzyme was sigmoidally dependent on the PEP concentration, indicating moderate positive co-operativity (Table 3), as is often found in allosteric enzymes. Although maximal PYK activity was detected in the presence of $\mathrm{Mg}^{2+}, \mathrm{Ni}^{2+}$ or $\mathrm{Co}^{2+}$ could substitute to some extent. No activity was detected in the absence of a divalent cation. The catalytic activity of PYK is controlled in nearly all organisms by one or more allosteric effectors. PYKs of bacterial origin are normally homotropically activated by PEP and the allosteric effectors fructose 1,6-bisphosphate (F1,6-BP), AMP and/or sugar monophosphates. Furthermore, most PYKs exhibit co-operativity and allosteric inhibition by ATP (Mattevi et al., 1996). As shown in Fig. 6(b), F1,6-BP, glucose 6-phosphate (G6-P) and AMP activated PYK, whereas ATP was inhibitory and ribose 5-phosphate exerted little effect.

PCK activity displayed an absolute dependence on the presence of divalent metal cations. Both $\mathrm{Mg}^{2+}$ and $\mathrm{Mn}^{2+}$ were required for maximal activity. When ATP and AMP were substituted for ADP, PCK activity was not detected. ATP and AMP strongly inhibited PCK activity in the presence of $4 \mathrm{mM}$ ADP (Fig. 6c). Moreover, 2oxoglutarate and glutamate also inhibited PCK activity (Fig. 6c). The bicarbonate concentration dependency of PCK activity deviated from Michaelis-Menten kinetics, being linear at concentrations of up to $140 \mathrm{mM}$. PCK 
Table 3. Biochemical properties of the PYC, PYK, PCK and MEZ of C. jejuni

\begin{tabular}{|llccc|}
\hline \multirow{2}{*}{ Enzyme } & Substrate & \multicolumn{3}{c|}{ Kinetic parameter measured: } \\
\cline { 3 - 5 } & & $K_{\mathrm{m}}(\mathrm{mM})$ & $\begin{array}{c}V_{\max }\left[\text { nmol } \text { min }^{-1}\right. \\
\left.(\mathbf{m g} \text { protein })^{-1}\right]\end{array}$ & Hill coefficient \\
\hline PYC & & 46 & NA \\
& Pyruvate & $0 \cdot 24$ & 88 & NA \\
PYK & ATP & $1 \cdot 76$ & 52 & NA \\
& Bicarbonate & $0 \cdot 64$ & 1227 & $1 \cdot 30$ \\
PCK & PEP & $0 \cdot 15$ & 1036 & NA \\
MEZ & ADP & $0 \cdot 27$ & 120 & NA \\
\hline
\end{tabular}

*NA, Not applicable.

observed hyperbolic kinetics when the initial velocity was plotted against PEP concentration (Table 3). Effector studies and kinetics for PCK were also performed on the pyk mutant and gave similar data (not shown) to those obtained for the wild-type strain, ruling out the possibility of interference of the PCK activity by PYK.

The MEZ activity of C. jejuni NCTC 11168 was strictly dependent on NADP; no activity was detected with NAD as a cofactor. Furthermore, no activity was measurable with D-malate. MEZ displayed sigmoidal kinetics when L-malate was the variable substrate. The affinity of the enzyme for malate was very low, with an apparent $K_{\mathrm{m}}$ of $8.97 \mathrm{mM}$ (Table 3 ). The Hill coefficient (Table 3) was indicative of positive co-operative interactions between the substrate and the enzyme. MEZ activity was demonstrated to be dependent on the presence of divalent metal cations. $\mathrm{Co}^{2+}, \mathrm{Ni}^{2+}$ or $\mathrm{Mg}^{2+}$, but not $\mathrm{Ca}^{2+}$, could substitute for $\mathrm{Mn}^{2+}$. MEZ activity from several organisms has been shown to be stimulated by $\mathrm{NH}_{4}^{+}$and $\mathrm{K}^{+}$(Gourdon et al., 2000; Driscoll \& Finan, 1996). Similarly, the MEZ activity of C. jejuni was increased three- and twofold in the presence of $\mathrm{K}^{+}$and $\mathrm{NH}_{4}^{+}$, respectively. In addition, MEZ activity was not affected by glutamate or AMP, whereas ATP, PEP and F1,6-BP markedly inhibited activity. G6-P, on the other hand, enhanced MEZ activity (Fig. 6d).

\section{DISCUSSION}

In this study, we have examined the metabolic alterations that occur in C. jejuni as a consequence of the mutational inactivation of PYK and the anaplerotic enzymes PYC and MEZ. The diminished PYK activity observed in the $p y c A$ and $p y c B$ mutants is suggestive of negative control of PYK by pyruvate. Similarly, negative regulation of PCK activity by PEP is observed in the $p y k$ mutant. The reduced growth of the $p y c A$ mutant on pyruvate and on lactate, but not on malate, as compared to the wild-type clearly indicates a role for PYC during growth on pyruvate and lactate. Restoration of the growth of the $p y c A$ mutant on pyruvate by the addition of a low concentration of malate is clearly indicative of an anaplerotic function.

Bacterial and eukaryotic PYCs described to date are homotetramers $\left(\alpha_{4}\right)$ of $110-130 \mathrm{kDa}$, with the exception of the Pseudomonas citronellolis, Azotobacter vinelandii and Methanobacterium thermoautotrophicum PYCs, which have an $\alpha_{4} \beta_{4}$ structure. The $\alpha$ subunit $(65 \mathrm{kDa})$, encoded by $p y c B$, carries the biotin moiety and contains all of the catalytically active sites necessary for the PYC reaction. The $\beta$ subunit ( $45 \mathrm{kDa})$, encoded by $p y c A$, offers conformational stability within the enzyme's core (Goss et al., 1981; Mukhopadhyay et al., 1998). In contrast to the $\alpha_{4}$ PYCs, all of which require or are stimulated by acetyl-CoA, the $\alpha_{4} \beta_{4}$ enzymes are fully active in the absence of this effector (Cohen et al., 1979; Mukhopadhyay et al., 1998). The presence of two genes that encode the $65 \mathrm{kDa}$ and $54 \mathrm{kDa}$ subunits of PYC, the lack of a $65 \mathrm{kDa}$ biotinylated band in the $p y c B$ mutant and the inability of acetyl-CoA to activate the PYC of C. jejuni suggest that this enzyme is most likely to be of the $\alpha_{4} \beta_{4}$ type. The $\alpha_{4} \beta_{4}$ PYCs also appear to be insensitive to the other characteristic metabolic inhibitors described thus far for this enzyme, including Laspartate and glutamate which are potent inhibitors of some eukaryotic, bacterial and fungal PYCs (Mukhopadhyay \& Purwantini, 2000; Modak \& Kelly, 1995; Fier \& Suzuki, 1969). The presence of a PEP carboxylase, which is regulated by acetyl-CoA and Laspartate (Liao \& Atkinson, 1971; O’Brien et al., 1977), in organisms possessing $\alpha_{4} \beta_{4}$ PYCs is noteworthy. Moreover, PYC content in these organisms is a function of the carbon source used for growth (O'Brien et al., 1977; Scrutton \& Taylor, 1974). It seems reasonable to conclude, therefore, that 'fine' control is not involved in the regulation of pyruvate carboxylation in these species. C. jejuni, on the other hand, has only a single enzyme (PYC) with the capacity to catalyse net synthesis of OAA from a three-carbon precursor, and therefore might be expected to display markedly different regulatory properties. In accord with this supposition, the C. jejuni enzyme was found to be potently inhibited by L-aspartate and by glutamate, both of which could 
potentially be catabolized by aspartate aminotransferase to produce OAA, the product of the PYC reaction.

Almost all of the bacterial PYKs are activated by phosphorylated sugars and adenosine mono- or diphosphates, the latter of which are indicative of a low cellular energy charge. F1,6-BP, the product of the reaction catalysed by 6-phosphofructokinase, allosterically regulates PYK via feed-forward activation, whereas ATP, the product of the PYK reaction itself, regulates via feed-back inhibition. The PYK of C. jejuni NCTC 11168 is typical of a glycolytic enzyme with respect to its activation by F1,6-BP, G6-P and AMP, and its inhibition by ATP. Moreover, the higher specific activity of PYK compared to that of PYC, PCK and MEZ is notable. These findings are surprising in view of the fact that C. jejuni does not catabolize glucose, as reported by Smibert (1984). Determination of glucose utilization using nuclear magnetic resonance assays of culture supernatants has confirmed the inability of $C$. jejuni to metabolize glucose (J. Velayudhan, S. Hall and D. J. Kelly, unpublished data). This may be attributed to the absence of 6-phosphofructokinase, which is a key regulatory enzyme for sugar degradation via the Embden-Meyerhof pathway (Fig. 1). The role of PYK in C. jejuni could be to metabolize an as yet unidentified substrate in a pathway that bypasses 6-phosphofructokinase, allowing for ATP synthesis by substratelevel phosphorylation. Substrates of the pentose phosphate pathway are one possibility. The presence of homologues for the non-oxidative branch of the pentose phosphate pathway in the C. jejuni genome could allow growth on pentoses. However, neither ribose, xylose nor arabinose was capable of supporting the growth of C. jejuni in MEM $\alpha$ (J. Velayudhan and D. J. Kelly, unpublished data). In congruence with the activation of the PYK of C. jejuni by F1,6-BP, primary sequence comparisons reveal that residue 403 of the PYK is a serine. In non-regulated PYKs this residue is strictly conserved as a glutamic acid, which may lead to ineffective binding of F1,6-BP (Jurica et al., 1998). Furthermore, residues Thr378-Ser-Ser-Gly-Lys-Ser383 in the PYK of C. jejuni correspond to a well-defined phosphate-binding pocket in the yeast PYK, at which the $6^{\prime}$-phosphate of F1,6-BP makes a series of hydrogen bonds to the side chains of these residues.

The inability to construct a $p c k A$ mutant and the detrimental effect of reduced PCK activity on the growth of the polar $p y c B$ mutant indicates that PCK activity is indispensable for the growth of C. jejuni. The paucity of alternative enzymes that can function in the capacity of PEP synthesis, as indicated by enzyme assays, suggests that OAA decarboxylation by PCK is crucial for precursor synthesis for anabolic pathways that leave central metabolism upstream of PEP. The direction in which PCK operates is probably determined by the ratio of adenine nucleotide pools and the steady-state concentrations of OAA and PEP, hence the sensitivity of the $C$. jejuni PCK to adenosine phosphates. In addition, higher intracellular PEP pools may account for the decreased PCK activity observed in the pyk mutant. The inability of the $p y c B$ mutant, which possesses low levels of PCK, to grow on malate as a sole carbon source is consistent with this enzyme functioning predominantly as a gluconeogenic OAA decarboxylase. Bacillus subtilis and E. coli pck mutants are, similarly, unable to grow on CAC intermediates, although they grow normally on glucose (Diesterhaft \& Freese, 1973; Goldie \& Sanwal, 1980). The carboxylation reaction was not saturable at bicarbonate concentrations up to $140 \mathrm{mM}$, precluding a role for PCK in PEP carboxylation, as the organism is unlikely to encounter such high bicarbonate concentrations under physiological conditions.

A role in NADPH generation for biosynthesis is a possible function for MEZ, similar to the MEZs of Corynebacterium glutamicum and Aspergillus nidulans (Gourdon et al., 2000; Wynn \& Ratledge, 1997). However, the very low affinity of the MEZ of Campylobacter jejuni for malate and the comparable phenotype of the mez mutant to that of the wild-type is suggestive of a minor anaplerotic role for this enzyme in C. jejuni.

\section{ACKNOWLEDGEMENTS}

This work was supported by a grant from the BBSRC to D.J.K for J.V. We gratefully acknowledge the technical assistance of S. Hall.

\section{REFERENCES}

Best, E. A. \& Knauf, V. C. (1993). Organization and nucleotide sequences of the genes encoding the biotin carboxyl carrier protein and biotin carboxylase protein of Pseudomonas aeruginosa acetyl coenzyme A carboxylase. J Bacteriol 175, 6881-6889.

Cohen, N. D., Duc, J. A., Beegen, H. \& Utter, M. F. (1979). Quaternary structure of pyruvate carboxylase from Pseudomonas citronellolis. J Biol Chem 254, 9262-9269.

Diesterhaft, M. D. \& Freese, E. (1973). Role of pyruvate carboyxlase, phosphoenolpyruvate carboxykinase, and malic enzyme during growth and sporulation of Bacillus subtilis. J Biol Chem 248, 6062-6070.

Driscoll, B. T. \& Finan, T. M. (1996). $\mathrm{NADP}^{+}$-dependent malic enzyme of Rhizobium meliloti. J Bacteriol 178, 2224-2231.

Ferrero, R. L., Cussac, V., Courcoux, P. \& Labigne-Roussel, A. (1992). Construction of isogenic urease-negative mutants of Helicobacter pylori by allelic exchange. J Bacteriol 174, 4212-4217.

Fier, H. A. \& Suzuki, I. (1969). Pyruvate carboxylase of Aspergillus niger: kinetic study of a biotin containing carboxylase. Can J Biochem 47, 697-710.

Goldie, A. H. \& Sanwal, B. D. (1980). Genetic and physiological characterisation of Escherichia coli mutants deficient in phosphoenolpyruvate carboxykinase activity. J Bacteriol 141, 1115-1121.

Gornicki, P., Scappino, L. A. \& Haselkorn, R. (1993). Genes for two subunits of acetyl coenzyme A carboxylase of Anabaena sp. strain PCC 7120: biotin carboxylase and biotin carboxyl carrier protein. J Bacteriol 175, 5268-5272.

Goss, J. A., Cohen, N. D. \& Utter, M. F. (1981). Characterisation of the subunit structure of pyruvate carboxylase from Pseudomonas citronellolis. J Biol Chem 256, 11819-11825.

Gourdon, P., Baucher, M.-F., Lindley, N. D. \& Guyonvarch, A. (2000). Cloning of the malic enzyme gene from Corynebacterium 
glutamicum and role of the enzyme in lactate metabolism. Appl Environ Microbiol 66, 2981-2987.

Jurica, M. S., Mesecar, A., Heath, P. J., Shi, W., Nowak, T. \& Stoddard, B. L. (1998). The allosteric regulation of pyruvate kinase by fructose-1,6-bisphosphate. Structure 6, 195-210.

Kelly, D. J. (2001). The physiology and metabolism of Campylobacter jejuni and Helicobacter pylori. J Appl Microbiol 90, $16 \mathrm{~S}-24 \mathrm{~S}$

Ketley, J. M. (1997). Pathogenesis of enteric infection by Campylobacter. Microbiology 143, 5-21.

Li, S.-J. \& Cronan, J. E., Jr (1992). The gene encoding the biotin carboxylase subunit of Escherichia coli acetyl-CoA carboxylase. J Biol Chem 267, 855-863.

Liao, C.-L. \& Atkinson, D. E. (1971). Regulation of the phosphoenolpyruvate branchpoint in Azotobacter vinelandii: phosphoenolpyruvate carboxylase. J Bacteriol 106, 31-36.

Marmur, J. (1961). A procedure for the isolation of deoxyribonucleic acid from microorganisms. J Mol Biol 3, 208-218.

Mattevi, A., Bolognesi, M. \& Valentini, G. (1996). The allosteric regulation of pyruvate kinase. FEBS Lett 389, 15-19.

Modak, H. V. \& Kelly, D. J. (1995). Acetyl-CoA-dependent pyruvate carboxylase from the photosynthetic bacterium $R$ hodobacter capsulatus: rapid and efficient purification using dyeligand affinity chromatography. Microbiology 141, 2619-2628.

Mukhopadhyay, B. \& Purwantini, E. (2000). Pyruvate carboxylase from Mycobacterium smegmatis: stabilisation, rapid purification, molecular and biochemical characterisation and regulation of the cellular level. Biochim Biophys Acta 1475, 191-206.

Mukhopadhyay, B., Stoddard, S. F. \& Wolfe, R. S. (1998). Purification, regulation, and molecular and biochemical characterisation of pyruvate carboxylase from Methanobacterium thermoautotrophicum strain $\Delta \mathrm{H}^{*}$. J Biol Chem 273, 5155-5166.

O'Brien, R. W., Chuang, D. T., Taylor, B. L. \& Utter, M. F. (1977). Novel enzymic machinery for the metabolism of oxaloacetate, phosphoenolpyruvate, and pyruvate in Pseudomonas citronellolis. J Biol Chem 252, 1257-1263.

Parkhill, J., Wren, B. W., Mungall, K. \& 18 other authors (2000). The genome sequence of the foodborne pathogen Campylobacter jejuni reveals hypervariable sequences. Nature 403, 665-668.

Sambrook, J., Fritsch, E. F. \& Maniatis, T. (1989). Molecular Cloning: a Laboratory Manual, 2nd edn. Cold Spring Harbor, NY: Cold Spring Harbor Laboratory.

Scrutton, M. C. \& Taylor, B. L. (1974). Isolation and characterisation of pyruvate carboxylase from Azotobacter vinelandii OP. Arch Biochem Biophys 164, 641-654.

Smibert, R. M. (1984). Genus Campylobacter Sebald and Véron 1963, 907 ${ }^{\mathrm{AL}}$. In Bergey's Manual of Systematic Bacteriology, vol. 1, pp. 111-117. Edited by N. R. Krieg \& J. G. Holt. Baltimore, MD: Williams \& Wilkins.

Tauxe, R. V. (1992). Epidemiology of Campylobacter jejuni infections in the United States and other industrialized nations. In Campylobacter jejuni: Current Status and Future Trends, pp. 9-19. Edited by I. Nachamkin, M. J. Blaser \& L. S. Tompkins. Washington, DC: American Society for Microbiology.

van Vliet, A. H. M., Wooldridge, K. G. \& Ketley, J. M. (1998). Ironresponsive gene regulation in a Campylobacter jejuni fur mutant. J Bacteriol 180, 5291-5298.

Wang, Y. \& Taylor, D. E. (1990). Chloramphenicol resistance in Campylobacter coli: nucleotide sequence, expression, and cloning vector construction. Gene 94, 23-28.

Wynn, J. P. \& Ratledge, C. (1997). Malic enzyme is a major source of NADPH for lipid accumulation by Aspergillus nidulans. Microbiology 143, 253-257.

Received 14 August 2001; revised 19 October 2001; accepted 5 November 2001 